\title{
Stray Animal Population Control: Methods, Public Health Concern, Ethics, and Animal Welfare Issues
}

\author{
Abubakar Abdulkarim, Mohd Azam Khan Bin Goriman Khan", and Erkihun Aklilu \\ Faculty of Veterinary Medicine, Universiti Malaysia Kelantan, 16100 Pengkalan Chepa, Locked Bag 36, Kota Bharu, Kelantan, Malaysia \\ *Corresponding author's Email: azamkhan@umk.edu.my; (DORCiD: 0000-0001-5282-435X
}

\begin{abstract}
Stray animal overpopulation has become one of the most serious global problems with many negative impacts on the community, environment, and public health. Most of the stray animals do not depend on humans for food and shelter, and therefore, can reproduce uncontrollably. The uncontrolled reproduction of stray animals increases their population that leads to a higher chance of predation, road traffic accidents, transmission of zoonotic diseases, and therefore, becoming vectors for some diseases. There are several methods for stray animal population control depending on the situation and the nature of the stray animals. These methods include contraceptives, trap-neuterreturn, poisoning, euthanasia, and gun shooting among others. Each of the outlined methods has its advantages and disadvantages as to their practicality, ease of conducting, cost, effectiveness, ethics, and animal welfare issues. In conclusion, to achieve successful control measures of the stray animal population and the problem they create, the concerned authorities need to design and enact animal rights laws, provide medical care (treatment and vaccination), feeding, shelter for the animals, and control their reproduction. Public health and environmental agencies may improve the services by regulating personal and environmental hygiene, prevention, and control of zoonotic and transmissible diseases that can be transmitted from stray animals to the public and other livestock respectively.
\end{abstract}

Keywords: Animal welfare, Ethics, Population control methods, Public health, Stray animals

\section{INTRODUCTION}

Stray animals are un-owned domestic animals, particularly pets, such as cats and dogs (Sandøe et al., 2019). They mostly live independently, reproduce uncontrollably, overpopulate the environment, and often become a source of nuisance and global challenge to public health and environmental safety (Voslářová and Passantino, 2012). There have been many debates on the nature of stray animals especially animals' welfare and their problems in the community, such as hunting behavior, nuisance, and predating other livestock and poultry farms (Crowley et al., 2019).

It has been estimated that approximately 7.6 million pet animals enter into stray every year, of which about 3.4 million are cats (Statistics - National Kitten Coalition, 2020). According to the World Health Organization (WHO), the estimated number of stray dogs was approximately 200 million or more worldwide between 2009 and 2010 (Patti, 2011). With this number of strayed animals, four to five million are euthanized every year (Fournier and Geller, 2004). The abandoned pet animals neither affect their welfare nor pose a significant risk to public health and the environment. This condition makes them vulnerable to starvation, road transport accidents, diseases, and even death. Under some conditions, they may also harm the environment and native species through competition, predation, and spreading infectious diseases (Jessup, 2004). It has been observed that the stray animals in some communities are catered to by the residents who take care of and provide food and shelter for them (Centonze and Levy, 2002).

Animal professionals, such as veterinary doctors, animal scientists, and animal welfare officers have considered pet animal overpopulation as a people problem rather than an animal problem (Gerhold and Jessup, 2013). For the achievement of effective management of stray animals in an environment, there is a need to understand the relationship between the population of the strayed aminal and their impacts on a different dimension of the environments, and to know the way their relationship will be affected by other menace and environmental factors (such as climates) (Legge et al., 2017). It is difficult to estimate the number of stray animals in a community due to their high reproductive capacity. Under normal conditions, animals like dogs and cats with their puppies and kitten can make a population of more than a hundred in a year, this means that despite high mortality rates, their population can still be sustained (Stoskopf and Nutter, 2004). Highly adaptable, these animals thrive in environments ranging from villages up to metropolitan urban cities.

Different methods have been used to control stray animal populations for several years. These methods include poisoning, conducting Trap-Neuter-Return (TNR) programs, performing euthanasia using a high dose of anesthetic agents, such as a barbiturate, and administering some hormone-based contraceptive agents, such as progesterone (progestin) and testosterone propionate. Each of the outlined methods has its own advantages and disadvantages in terms 
of their practicality, ease of conducting, cost, effectiveness, ethics, and animal welfare issues. Therefore, this literature review summarizes the impact of stray animals on the environment, the effect on the community and public health concern, and methods of stray animal population control with the ethics and animal welfare issues related to the different methods of stray animal population control.

\section{Stray animals}

Stray animals are free-roaming pet animals, especially dogs and cats that survive without human care and with an uncontrollable population increase. This condition leads to significant health and welfare problems for the animals concerning their public health and safety with environmental concerns (Fournier and Geller, 2004). Stray animals, such as dogs and cats, due to their predation nature may attract other wild predators, such as fox and coyotes, into society or community (Seimenis, 2004; Turner et al., 2012).

Stray animals are considered as one of the major public health problems worldwide in terms of their welfare and society. This concern mainly arises from the possible interactions of these strayed animals in many countries due to their interaction with people, especially children (Slater, 2001). Some may cause injuries to children through bites and scratches and may transmit zoonotic diseases. Interaction between humans and animals has been a common incidence over the past several centuries. Dogs play different roles in society, they act as pets, guards against thieves, crime detectors, runners, and hunters in champions, similarly, cats are also kept as pets and used for hunting rodents and reptiles that destroy properties at home (Bernstein, 2007).

It is essential to establish a long-term and sustainable approach to deal effectively with stray animal populations. The idea is to protect the people against those animals and safeguard the welfare and health of the animals. Experiences from the efforts in stray animal control show that effective control measures involve the adoption of more than one strategy. Among the strategies, western societies' concept of "ownership" is the most popular. This strategy focuses on a comprehensive, coordinated, and progressive program of owner education, mandatory registration and identification, environmental management, reproduction control of pets, and management of the animal population through regulated breeding and marketing (OIE, 2019). All these rules should be regulated by effective and enforced legislation. Implementing these rules successfully requires the cooperation and collaboration of more than one agency which in turn depends upon the willingness of government departments, municipalities, veterinary agencies, and non-government organizations (NGO's) to work together (Tasker, 2007).

\section{Source of stray animals}

In developing countries, many pet animals especially, cats and dogs, are abandoned on the streets and become part of a stray population. While in developed countries, stray cats and dogs are taken to animal shelters (Voslářová and Passantino, 2012).

Abandoned pet animals are increasing in number and have become a problem in many countries. Even though the proportion of the stray dog and cat population is not frequently measured, evidence shows that their numbers are increasing daily (OIE, 2019). The population density of the strayed animals may differ from the culture, habitat, and socio-economic conditions of the nation.

\section{Stray dogs}

Stray dogs are free-roaming dogs in communities that have become one of the major problems in society. They harm the environment and human health. Many studies have shown the important roles that pet animals are playing in the life of human being and dog is considered as one of the animals that kept as a pet in the past centuries (Ghasemzadeh and Namazi, 2015), but most of the pet dogs are going to stray due to owners cannot afford to take care of them. Many stray dogs roaming on the streets at night cause nuisance, defecate everywhere, scavenging on garbage, cause or get involved in road accidents, transmit diseases, and may die on the streets (Lyu, 2015). Stray dogs can cause an accident when running into the road, which might either result in injury to other people or themselves. Besides, stray dogs have become a threat to human lives and health by spreading deadly diseases, such as rabies. It had estimated by WHO that there are more than 200 million stray dogs worldwide, and every year 55000 people die from rabies transmitted by rabid dogs' bites (Chandran and Azeez, 2016).

Most of the dogs were considered as stray due to abandonment by their owners for some reason such as family immigration, diseases, and unbearable physiological behaviors of pets, no fund for treatment or feeding. Strayed dogs are facing many problems concerning their management. Sheltering alone cannot be an option for stray dog management because many conditions, such as feeding, disease treatment, annual vaccination and deworming, and appropriate quarantine require a lot of money to settle the conditions. In this regard, money and resources will never be sufficient to meet the sufficient demands for stray dog management (Lyu, 2015).

Dog population management (DPM) is an adaptable concept that aims to promote the health and well-being of stray dogs by minimizing the problems caused by the dogs while targeting to reduce their population size. Dog 
population management can be achieved for many animal welfares, economic purposes, public health, and safety. These may be regulated through eradicating the transmission of infectious diseases and zoonotic diseases (especially rabies) by controlling dog bites or injuries to human beings and other animals, minimizing the occurrence of road or transport accidents, and reducing nuisance and fecal contamination of the environment caused by stray dogs during breeding (Taylor et al., 2017). In this regard, DPM programs have some goals depending on the conditions which may or may not be permanently limiting the size of the stray dog population. The means to achieve DPM objectives are planned to make a long-term positive impact on stray dog populations, in comparison to dog culling (Jackman and Rowan, 2007).

\section{Stray cats}

Stray cats, also known as feral cats, are free-roaming unowned cats mainly independent (Turner et al., 2012). The Feral domestic cat is one of the most challenging invasive species worldwide. The stray cat population has contributed to the extinction of some species of birds, such as Socorro dove (Zenaida graysoni) and Manx shearwater (Puffinus puffinus) among others, mammals, and reptiles in the environment which represent about $14 \%$ of modern extinctions in these vertebrate groups (Razgūnaitè et al., 2019). Many stray cats are roaming along the street, sometimes seen in restaurants, institutions, places of worship, and refuse dump looking for food. The same problem has also been observed in other developing countries (Legge et al., 2017).

It is difficult to estimate the number of stray cats in a community due to their high reproductive capacity. Cats are sexually mature by 5 to 6 months of age. Therefore, even with high mortality rates cat population can be sustained (Stoskopf and Nutter, 2004). The reproductive approaches, such as early sexual maturity, seasonally polyestrous cycling, and multiparous pregnancies, contribute to the prolific nature of the cat population (Kutzler, 2007). Highly adaptable, cats have thrived in environments ranging from villages, towns, and metropolitan urban cities (Legge et al., 2017).

Currently, the rate of cat eradication from society does not indicate significant changes in many societies where invasive cats are likely to challenge native wildlife (Medina et al., 2011). Therefore, to expand their biodiversity benefits, a new approach to cat eradication needs to be designed and implemented effectively. Furthermore, conservation biologists have a good understanding of and play an important role in the way stray cats impact native species of the environment (Medina et al., 2011).

\section{Effect of stray animals on the community}

The life activities of domestic animals mainly depend on their owners, which can be in form of personal ownership or a group of people in a community. Owners play an important role in the community or environments by regulating the management of pets (Taylor et al., 2017). In many cases, some of the owners may not afford to take care of the animals. These may pose any risk to the communities since they cannot maintain the well-being of the animals.

Due to the above conditions, pet animals are abandoned while roaming freely and join the stray population in the community and become a common source of spread of diseases to humans (zoonotic) and other pet animals across the community (Robertson, 2008). The main challenges and effects associated with stray animals on the community include predation, road traffic accident, defecation, urine spraying, nuisance, running and fighting during breeding, scavenging on open garbage and scattering all sealed refused, attacking people as in case of a rabid dog, soiling and threatening pet animals by attacking and biting (Trotman and Brown, 2007).

\section{Stray animals and public health}

Public health as a field of medicine is important because it comprises training in many fields and experience of working in teams with familiarity with interdisciplinary research. Stray animals are the major cause of public health problems and animal welfare concerns in any part of the world. Abandoned animals living in mass can reproduce and increase the number of the new stray animal population (Seimenis and Tabbaa, 2014). Public health plays an important role in minimizing stray animal overpopulation by controlling their reproduction, improving their health and well-being by vaccinating and treatment of sick ones among the animals, and increasing the responsibility by providing ownership to the animals.

The impact of public health on the occurrence of stray animals in both rural and urban areas includes the emergence and endemicity of many zoonotic diseases. Disease transmission from stray animals to humans and livestock depends on the nature of the countries, the climatic regions, and the health status and density of the human population (Robertson, 2008). Public health and epidemiology as disciplines are essential in understanding the impact of the stray animal population. The field of public health and epidemiology is critical in understanding the demographics, potential interventions, types of the problems, and required assessment of stray animal population control (Slater, 2001).

The Public health program will design control measures on stray animal populations and the spread of zoonotic diseases among the human population. These can be through public awareness of the harmful existence of stray animals in a society, public and environmental hygiene, controlling vectors that transmit the causative agents of the diseases, educating the public on zoonotic diseases, and performing measures to prevent and control the disease. The zoonotic 
diseases of public health importance that can transmit to humans via stray animals include brucellosis, toxoplasmosis, rabies, campylobacteriosis, leishmaniasis, bartonellosis, salmonellosis, Pasteurellosis, and leptospirosis with others (Ghasemzadeh and Namazi, 2015).

The stray animal population control can be achieved by applying the aims of public health to determine why the animals are on strayed? Where are their sources? What are their populations? What are the problems they cause to people and livestock? Which way to evaluate and intervene in the situation and what are the costs to confront the situations (Slater, 2001)?.

\section{Impact of stray animals on the environment}

The impact of stray animals, especially dogs and cats, is more visible than other pet animals concerning the problems they create in the environment (Trotman and Brown 2007). Some species become tamed without showing fear of contact with humans and other domestic animals, which gives the chance of becoming the source of causing an effect on the environment and spread of infectious diseases (Seimenis and Tabbaa, 2014).

Stray animals also play an important role in transmitting diseases to humans and domestic animals through environmental pollution. These issues happen due to poor environmental hygiene and either directly from contact with feces (Traversa et al., 2014), urine, and other excretions from stray animals, or indirectly via infected or contaminated foods and food products obtained from diseased animals such as milk, and poorly prepared meat and meat products (Seimenis, 2004). Some Stray dogs and cats are scavenging around slaughterhouses for feeding, which may serve as an important source of disease transmission. In this regard, besides the control of scavenging stray animals, strict measures are to be taken by providing incinerators or disposal sites to prevent their access to the sources of infections, particularly the abattoirs and butcheries (Seimenis and Tabbaa, 2014).

Stray animals can become opportunistic predators (Medina et al., 2011) by attacking small animals, such as lizards, rats, and birds, and also by attracting other wild predators, such as fox, jackals, and coyotes into the environment (Turner et al., 2012) which can spread the impacts of predation across the environment (Dickman and Newsome, 2015). In an initial way, that was not the natural habitat of the ecological equations.

\section{Ethics and animal welfare issues related to stray animal population control}

To address the ethical issues and welfare of stray animals, there is a need to consider some factors, such as feeding, shelter, health care, and in some instant, their interaction with people (Briggs, 2013). The caretakers are expected to show sympathy and ethical concerns by providing the necessary care for the animals (Centonze and Levy, 2002). Neutering of stray animals improves their health and body score, minimizes roaming, and becomes friendly. It also observed that some offsprings of strayed animals die or disappear within some months of birth with trauma from children or other stray animals, starvation, disease, and mobile accident (Stoskopf and Nutter, 2004). Such conditions depend on geographical locations and other factors including weather and human population density. It is observed that the mortality rate of some stray animals was high, and the causes of death ranged from mobile accidents, poisoning, disease conditions, and attack from other stray animals that correspond with a poor standard of life (Aguilar et al., 2015).

The welfare of stray animals can vary noticeably. Some communities consider the elimination of stray animals as humane than allowing them to live a miserable life. Humanely considering stray animal population control, one method does not fit all the conditions due to their differences (Stoskopf and Nutter, 2004). Campaigning to educate and enlighten the public on domestic animal ownership responsibilities and their effects and implications of abandoning should be highlighted routinely (Param, 2016). Animal welfare agencies are the agencies that care and concern about the health and well-being of animals (domestic, wild, and stray). Some of these agencies include the American Society for the Prevention of Cruelty to Animals (ASPCA), Humane Society of the United States (HSUS) (Rowan and Kartal, 2018), International Fund for Animal Welfare (IFAW), Animal Welfare Institute, Animal Welfare Organization, Ipoh Society for the Prevention of Cruelty to Animals (ISPCA) (Param, 2016), World Animal Protection (WAP), International Animal Rescue, Global Alliance for Rabies Control, National Animal Welfare Trust among others. These agencies aim to control stray animal populations through enacting and enforcing appropriate policies that regulate humane animal population control and provide animal-control facilities. These include sterilization of all adopted animals. And the procedures require proper training, surveillance, and dedication from the staff and volunteers (Fournier and Geller, 2004).

The ethical approach for stray animal population control from both human and animal welfare points of view. The human welfare view usually focuses on the spread of zoonotic diseases and other effects caused by stray animals. The animal welfare view rests on the expectation that disease and death in uncontrolled populations of strayed animals cause suffering that prevails over the associated control programs (Kathy, 2006). These opinions are influenced by different views about the fundamental values related to suffering, freedom, and the existence of stray animals. The most used ethical way of resolving this issue is either by the humane killing of strayed animals (mostly euthanasia) or by controlling their reproduction (sterilization or neutering, OIE, 2019). 


\section{Methods of controlling the population of stray animal \\ Contraception}

Contraception is the method used to prevent the occurrence of pregnancy in female animals and can be achieved through the use of a medication, device, or procedure. Choices of contraceptive methods should address factors, such as efficacy, safety, availability, acceptance, and affordability (Bansode et al., 2019). Contraceptive agents can be used in different forms depending on the types (pills or injectable forms) and the method of use. Some contraceptive agents induce contraception by altering the hormonal activities, especially the hormone-based contraceptive like diethylstilbestrol, progestin, medroxyprogesterone acetate (MPA), proligestone (PROL), and mibolerone (Asa, 2018). While the use of implants is impaired through the use of an intrauterine device (IUD), such as the Depo-proveras and melengestrol acetate (MGA) implants (Boutelle and Bertschinger, 2010).

The use of the non-surgical contraceptive method is more accurate when owners request to breed their animals in the future (Massei and Miller, 2013) unless in the case of some diseases, such as rabies, where permanent sterilization is preferable. Most of the contraception techniques should be implemented by trained veterinarians, except for oral contraceptives. Most of the new devices are not licensed and require expert and trained personals to use them, and their cost is very high (Taylor et al., 2017).

Female animals treated with hormone-based contraceptives may experience some side effects, such as hormonal in-balance, uterine infection, pyometra, mammary tumor, diabetes mellitus, and other potentially lifethreatening side effects that may arise from the drugs. Therefore, animals treated with hormone-based contraception require daily monitoring by veterinarians for the prevention of the mentioned complications (Asa, 2018).

The advantages of contraception include a longer duration before reverse, fewer side effects, suppression of sexual behavior, easy administration, a low cost, and also applicable for humans (Cathey and Memon, 2010). On the other hand, the disadvantages of this method are the need for repetition, quick reverse when discontinued using the repeated dose, possible slow onset of activity in some drugs, possible chance of a reaction at the injection site (Cathey and Memon, 2010).

\section{Trap-neuter-return}

Stray animal control population must require safety considerations for non-target animals and humans, be supported by municipal agencies or charitable organizations, including plans to reduce continuous stray dog and cat migration and reproduction, and be aesthetically acceptable to the public (Levy et al., 2003).

The considerable argument concerning the methods of controlling stray animals, particularly identifying the alternatives that are practicable, effective, and humane, TNR programs are designed to pause reproduction without causing harm to the animals (Scott et al., 2002).

In this method, animals are trapped, neutered, and returned to their previous habitat. The procedure is performed by veterinary surgeons through spaying the female animal or castration in a male animal. The concept of TNR as a humane method for stray animal population control is recommended by welfare organizations (Centonze and Levy, 2002). However, this method requires a regular surveillance and maintenance program that can be costly. The expenses of the TNR method depend on the level of resources that the stakeholders execute for the management of the problems.

Mostly, the ongoing costs of stray animal control through the TNR method will be high. The technique is expensive, inappropriate for large-scale implementation, and time-consuming, as well as requiring many expert veterinary surgeons (DEWHA, 2008). Other disadvantages of this method that may be encountered, in case of poor management after the surgery, there's a chance of some complications such as urinary incontinence and there will be an increase in the incidence of cystic, hematologic, and osteo-tumors (Scott et al., 2002). However, it has many advantages, such as producing complete sterilization, minimizing the incidence of sexual behaviors, and reducing the incidence of mammary, ovarian, and uterine tumors (Levy et al., 2003).

\section{Euthanasia}

The word euthanasia derived from a Greek called "safe killing" or "good death" is a humane method of killing animals through medical measures. It can achieve by administering a large volume of anesthetic agents such as barbiturates (Materstvedt et al., 2003), ketamine, a compound containing potassium (Harris, 2001) beyond accurate dosage. It is painless and induces rapid unconsciousness followed by respiratory and cardiac arrest, and finally causes death. In other words, it is the painless inducement of a quick death. The World Society for the Protection of Animals (WSPA) advocates that the killing of pet animals through euthanasia is a humane method and should be carried out only by trained professionals. The organization also accepts that euthanasia is humane and acceptable if the animal facing the hardship of life due to illness, injury, or behavioral problems, but WSPA avoided the use of euthanasia in healthy animals and considered it as cruelty (Veikune, 2014).

This method costs very high in many respects, concerning financing the officials that handle the activities, professionals that perform the euthanasia, the ideal way of disposing of the carcasses, and the workers that disposing of the carcasses. For many years, euthanasia has been desired as an alternative to deal with the overpopulation of pet 
animals (Purswell and Kolster, 2006). Regardless of the technique used, it is good to reduce distress, anxiety, and pain by ensuring that the person who performs the euthanasia is well trained. Some of the euthanasia must use in combination with sedation before euthanizing the animal. The chemical agents used for euthanasia include Chloral hydrate, Formalin, Chloroform, Nitrous oxide, Ether, pentobarbital, and some neuromuscular blocking agents such as nicotine, potassium chloride, and magnesium sulfate (OIE, 2019). Based on the above conditions, euthanasia is a challenging method and is not suitable for controlling the stray animal population. It may help in reducing their populations, but should not be considered as an active measure for the control of the stray animal population. Besides, euthanasia is necessary for old age, seriously injured, and dying pets suffering from severe pain, but not suitable for healthy animals (Bartlett et al., 2005).

The advantages of euthanasia (OIE, 2019) as a method of control for stray animal populations include, it induces painless death without cruelness, not like poisoning, it is accepted ethically worldwide, the chemical agents are of low cost, and minimal materials are required.

Regarding the disadvantages, this method must be performed by an expert (OIE, 2019), is difficult for a large population, requires some chemical agents which are not always available, demands agents like barbiturate that have long withdrawal action may cause sedation or other complications to any predator that fed on the euthanized carcass, needs a large volume of the chemical agents for inducing euthanasia which may be expensive to perform (Purswell and Kolster, 2006).

\section{Poisoning}

Poisons are chemical substances that cause damages or harm to the organs, the system, or the body. It happens due to chemical reactions occurring when an animal absorbs or is exposed to the chemicals by a sufficient quantity. Poisoning has been one of the methods for the control of the stray animal population. The most commonly used chemicals are anticoagulants and Sodium monofluoroacetate (Sherley, 2004). The major problem associated with poisoning is that it is cruel, and animals suffer a painful and slow death (Robertson, 2008). The clinical signs associated with poisoning include excessive salivation, vocalization, vomiting, snapping and biting, diarrhea, nystagmus, twitching of facial muscles, pulmonary congestion, tremor, tonic convulsions with paddling of the limbs, and finally death due to cardiac and respiratory arrest (Sherley, 2004). Regarding risks of poisoning, it may affect other non-targeted species that may directly or indirectly be exposed to the poison in the targeted environment. Likewise, there will be a risk of human exposure to the poison, especially children who are close to animals on playgrounds (Lyu, 2015), for whom there will be a chance of being exposed to the poison, for this reason, it is not an ideal way of practice and must be abandoned. However, poisoning as a method of stray animal population control can be advantageous since it is easy and efficient, cheaper, and can be used in a large population, it has less labor-intensive than other techniques (Mitchell, 2011). However, it causes painful death, it is cruel compared to other methods, there is a risk to other non-target animals and human beings, mainly children, it has animal welfare implications (Mitchell, 2011).

\section{Shooting}

Shooting is another method of stray animal population control (McLaughlina and Aitken, 2011) that is quick, painless, and efficient. It can be achieved if the bullets hit the targeted animals. There is a challenge of handling a gun by everyone without government permission. Shooting stray animals on the street is dangerous and has many risks if the target missed and mistakenly hits people (Mitrofanova et al., 2018). In other cases, some stray animals may not die on shooting and stay alive with pain and injury for long. These cases are mostly considered inhumane and brutal acts. (Lyu, 2015).

The use of shooting as a method of stray animal control is beneficial since it is considered humane if used on target animals, is target-specific, can be used as clean-up techniques, is the fastest method of controlling rabid dogs, and it can be applied to other animals with the same conditions (Lyu, 2015). However, it requires a skilled and trained person to shoot, is dangerous to people and non-targeted animals, needs proper equipment, and may damage the brain if the animal was shot by the head, especially as in case of a rabid dog where the brain is required for the rabid test, can cause severe wounds and pain if the animal does not die by shot instantly, and it needs government approval before carrying out the operation.

\section{CONCLUSION}

Stray animal overpopulation has become a global challenge that requires immediate solutions to manage their population. The most common methods that are used for stray animal population control include surgical neutering (sterilization) and non-surgical contraception techniques. The non-surgical contraception methods entail immunocontraceptives agents and hormone-based contraceptives, while the surgical methods of controlling stray animals are through spying and castration of female and male animals, respectively. Other available control methods are trap neuter return, euthanasia, poisoning, trap and relocate, and gun shooting among others. To achieve successful control measures of stray animal populations and the problems they cause in a community or environment, the 
concerned authorities have to design and enact animal rights laws. The law needs to address the feeding, shelter, medical care (treatment and vaccination), management, welfare, and legal rights of stray animals. To establish a prominent system of monitoring and management of the stray animal population, the community has to be educated about the problems that the animals are causing if they were on free-roaming and to provide a possible solution to the problem that may cause by the stray animals. Public health and environmental agencies may improve the services by regulating personal and environmental hygiene, prevention, control of zoonotic and transmissible diseases that can be transmitted from stray animals to the public and other livestock respectively.

\section{DECLARATION}

\section{Authors' contributions}

All authors contributed equally to this work.

\section{Competing interests}

The authors declare that they have no conflict of interest.

\section{Ethical consideration}

Ethical issues (including plagiarism, consent to publish, misconduct, double publication and/or submission, and redundancy) have been checked by the authors.

\section{Acknowledgments}

The authors gratefully acknowledged the effort of all who reviewed and provided a helpful comment on the manuscript. For the images used for the graphical abstract, the authors also acknowledged the attributions of images by Ingrid E. Newkirk, The Canadian Press, Patti Strand, and Healthy Homemade.

\section{REFERENCES}

Aguilar G, Farnworth M, and Winder L (2015). Mapping the stray domestic cat (Felis catus) population in New Zealand: Species distribution modelling with a climate change scenario and implications for protected areas. Applied Geography, 63: 146-154. DOI: https://www.doi.org/10.1016/j.apgeog.2015.06.019

Asa CS (2018). Contraception in dogs and cats. Veterinary Clinics of North America - Small Animal Practice, 48(4): 733-742. DOI: https://www.doi.org/10.1016/j.cvsm.2018.02.014

Bansode OM, Sarao MS, and Cooper DB (2019). Contraception. In StartPearls. StartPearls Publishing. Available at: https://www.ncbi.nlm.nih.gov/books/NBK536949/

Bartlett PC, Bartlett A, Walshaw S, and Halstead S (2005). Rates of euthanasia and adoption for dogs and cats in Michigan animal shelters. Journal of Applied Animal Welfare Science, 8(2): 97-104. DOI: https://www.doi.org/10.1207/s15327604jaws0802_2

Bernstein PL (2007). The human-cat relationship. Springer, pp. 47-89. Available at: https://link.springer.com/chapter/10.1007/978-1-4020-3227-1_3

Boutelle SM, and Bertschinger HJ (2010). Reproductive management in captive and wild canids: Contraception challenges. International Zoo Yearbook, 44(1): 109-120. DOI: https://www.doi.org/10.1111/j.1748-1090.2009.00107.x

Briggs J (2013). Contraception and fertility control in dogs and cats report. Available at: http://carodog.eu

Cathey M, and Memon MA (2010). Nonsurgical methods of contraception in dogs and cats: where are we now? Veterinary Medicine, 105 : 12-17. Available at: https://www.dvm360.com/view/nonsurgical-methods-contraception-dogs-and-cats-where-are-we-now

Centonze LA, and Levy JK (2002). Characteristics of free-roaming cats and their caretakers. Journal of the American Veterinary Medical Association, 220(11): 1627-1633. DOI: https://www.doi.org/10.2460/javma.2002.220.1627

Chandran R, and Azeez PA (2016). Stray dog menace: Implications and management. Economic and Political Weekly, 51(48): 58-65. Available at: https://www.epw.in/journal/notes/stray-dog-menace

Crowley SL, Cecchetti M, and McDonald RA (2019). Hunting behaviour in domestic cats: An exploratory study of risk and responsibility among cat owners. People and Nature, 1(1): 18-30. DOI: https://www.doi.org/10.1002/pan3.6

Department of the Environment, Water, Heritage and the Arts (DEWHA) (2008). Threat abatement plan for predation by feral cats. Department of the Environment, Water, Heritage and the Arts. Available at: http://www.environment.gov.au/biodiversity/threatened/tap-approved.html

Dickman CR, and Newsome TM (2015). Individual hunting behaviour and prey specialisation in the house cat Felis catus: Implications for conservation and management. Applied Animal Behaviour Science, 173: 76-87. DOI: https://www.doi.org/10.1016/j.applanim.2014.09.021

Fournier AK, and Geller SE (2004). Behavior analysis of companion-animal overpopulation: A conceptualization of the problem and suggestions for intervention. In Behavior and Social Issues, 13: 51-69. DOI: https://doi.org/10.5210/bsi.v13i1.35

Gerhold RW, and Jessup DA (2013). Zoonotic diseases associated with free-roaming cats. Zoonoses and Public Health, 60(3): 189-195. DOI: https://doi.org/10.1111/j.1863-2378.2012.01522.x

Ghasemzadeh I, and Namazi SH (2015). Review of bacterial and viral zoonotic infections transmitted by dogs. Journal of Medicine and Life, 8(4): 1-5. Available at: http://www.ncbi.nlm.nih.gov/pubmed/28316698

Harris NM (2001). The euthanasia debate. Journal of the Royal Army Medical Corps, 147(3): 367-370. DOI: https://www.doi.org/10.1136/jramc-147$\underline{03-22}$

Jackman J, and Rowan A (2007). Free-roaming dogs in developing countries. Animals, 8(5): 55-78. Available at: https://www.wellbeingintlstudiesrepository.org 
Jessup DA (2004). The welfare of feral cats and wildlife. Journal of the American Veterinary Medical Association, 225: 1377-1383. DOI: https://www.doi.org/10.2460/javma.2004.225.1377.

Kathy N (2006). 'Street dog' Population control case study. Vetwork - Resource Centre - Animal Health and Welfare. Available at: http://www.vetwork.org.uk/abc.htm

Kutzler MA (2007). Estrus induction and synchronization in canids and felids. Theriogenology, 68(3): 354-374. DOI: https://www.doi.org/10.1016/j.theriogenology.2007.04.014

Legge S, Murphy BP, McGregor H, Woinarski JCZ, Augusteyn J, Ballard G, and Zewe F (2017). Enumerating a continental-scale threat: How many feral cats are in Australia? Biological Conservation, 206: 293-303. DOI: https://www.doi.org/10.1016/j.biocon.2016.11.032

Levy JK, Gale DW, and Gale LA (2003). Evaluation of the effect of a long-term trap-neuter-return and adoption program on a free-roaming cat population. Journal of the American Veterinary Medical Association, 222(1): 42-46. DOI: https://www.doi.org/10.2460/javma.2003.222.42

Lyu P (2015). Proposal on solutions to stray dog problem in American cities. Journal of Political Sciences and Public Affairs, 3(3): 1-3. DOI: https://www.doi.org/10.4172/2332-0761.1000175

Trotman M, and Brown HJ (2007). Regional realities: Impact of stray dogs and cats on the community Impact on economy, including tourism impact on livestock, wildlife and the environment. Available at: https://www.hsi.org/wp-content/uploads/welfareresources/regional_realities.pdf

Massei G, and Miller LA (2013). Nonsurgical fertility control for managing free-roaming dog populations: A review of products and criteria for field applications. Theriogenology, 80: 829-838. DOI: https://www.doi.org/10.1016/j.theriogenology.2013.07.016

Materstvedt LJ, Clark D, Ellershaw J, Førde R, Boeck Gravgaard AM, Müller-Busch HC, and Rapin CH (2003). Euthanasia and physician-assisted suicide: A view from an EAPC Ethics Task Force. Palliative Medicine, 17(2): 97-101. DOI: https://www.doi.org/10.1191/0269216303pm673oa

McLaughlina EA, and Aitken RJ (2011). Is there a role for immunocontraception? Molecular and Cellular Endocrinology, 335: 78-88. DOI: https://www.doi.org/10.1016/j.mce.2010.04.004

Medina FM, Bonnaud E, Vidal E,Tershy BR, Zavaleta ES, Donlan CJ, Keitt BS, Corre ML, Horwath SV, and Nogales M (2011). A global review of the impacts of invasive cats on island endangered vertebrates. Global Change Biology, 17(11): 3503-3510. DOI https://www.doi.org/10.1111/j.1365-2486.2011.02464.x

Mitchell J (2011). Poisoning of feral pigs. NQ Dry Tropics, Townsville. Available at: https://sugarresearch.com.au/sugar_files/2017/02/Poisoning_of_feral_pigs.pdf

Mitrofanova SY, Fedorchuk KV, Grigoryeva EV, Buslaev SI, Kurbanov RA, Gurbanov RA, and Belyalova AM (2018). Student environmental attitude towards stray domestic animals. Ekoloji, 27(106): 1895-1902. Available at: http://www.ekolojidergisi.com

Param S (2016). Need to look at humane ways to reduce the stray animals situation. Available at: https://www.malaysiakini.com/letters/360169

Patti S (2011). The Global Stray Dog Population Crisis. National Animal Interest Alliance. Retrieved November 14, 2020. Available at: http://www.naiaonline.org/articles/the-global-stray-dog-population-crisis-and-humane-relocation\#sthash.O9Kc3L4P.x1QzPm3O.dpbs

Purswell BJ, and Kolster KA (2006). Immunocontraception in companion animals. Theriogenology, 66: 510-513. DOI: https://www.doi.org/10.1016/j.theriogenology.2006.04.018

Razgūnaitė M, Radzijevskaja J, Sabūnas V, Karvelienė B, and Paulauskas A (2019). Vector-borne zoonotic pathogens in cats. Biologija, 65(2): 140149. DOI: https://www.doi.org/10.6001/biologija.v65i2.4030

Robertson SA (2008). A review of feral cat control. Journal of Feline Medicine and Surgery, 10(4): 366-375. DOI: https://www.doi.org/10.1016/j.jfms.2007.08.003

Rowan A, and Kartal T (2018). Dog population and dog sheltering trends in the United States of America. Animals, 8: 68. DOI: https://www.doi.org/10.3390/ani8050068

Sandøe P, Jensen JBH, Jensen F, and Nielsen SS (2019). Shelters reflect but cannot solve underlying problems with relinquished and stray animals-A retrospective study of dogs and cats entering and leaving shelters in Denmark from 2004 to 2017. Animals, 9(10): 1-14. DOI: https://www.doi.org/10.3390/ani9100765

Scott KC, Levy JK, and Crawford PC (2002). Characteristics of free-roaming cats evaluated in a trap-neuter-return program. Journal of the American Veterinary Medical Association, 221(8): 1136-1138. DOI: https://www.doi.org/10.2460/javma.2002.221.1136

Seimenis A, and Tabbaa D (2014). Stray animal populations and public health in the South Mediterranean and the Middle East regions. Veterinaria Italiana, 50(2): 131-136. DOI: https://www.doi.org/10.12834/VetIt.48.134.3

Seimenis AM (2004). Urban veterinary hygiene in developing countries. The 29th Congress of the World Small Animal Veterinary Association. Available at: https://www.vin.com/apputil/content/defaultadv1.aspx?pId=11181

Sherley M (2004). The traditional categories of fluoroacetate poisoning signs and symptoms belie substantial underlying similarities. Toxicology Letters, 151: 399-406. DOI: https://www.doi.org/10.1016/j.toxlet.2004.03.013

Slater MR (2001). The role of veterinary epidemiology in the study of free-roaming dogs and cats. Preventive Veterinary Medicine, 48(4): 273-286. DOI: https://www.doi.org/10.1016/S0167-5877(00)00201-4

Statistics - National Kitten Coalition (2020). Available at: https://kittencoalition.org/news-events/statistics/

Stoskopf MK, and Nutter FB (2004). Analyzing approaches to feral cat management-one size does not fit all. Journal of the American Veterinary Medical Association, 225: 1361-1364. DOI: https://www.doi.org/10.2460/javma.2004.225.1361

Tasker L (2007). Stray animal control practices (Europe). World Society for the Protection of Animals (WSPA) and the Royal Society for the Prevention of Cruelty to Animals International (RSPCA International) Report. Available at: http://www.stray-afp.org

Taylor LH, Wallace RM, Balaram D, Lindenmayer JM, Eckery DC, Mutonono-Watkiss B, and Nel LH (2017). The role of dog population management in rabies elimination-A review of current approaches and future opportunities. Frontiers in Veterinary Science, 4: 1-15. DOI: https://www.doi.org/10.3389/fvets.2017.00109

Traversa D, Frangipane Di Regalbono A, Di Cesare A, La Torre F, Drake J, and Pietrobelli M (2014). Environmental contamination by canine geohelminths. Parasites and Vectors, 7: 1-9. DOI: https://www.doi.org/10.1186/1756-3305-7-67

Turner P, Berry J, and Macdonald S (2012). Animal welfare bien-être des animaux animal shelters and animal welfare: Raising the bar. Canadian Veterinary Journal, 53: 893-896. Available at: https://pubmed.ncbi.nlm.nih.gov/23372200/

Veikune C (2014). Companion animal overpopulation on o'ahu. Available at: https://scholarspace.manoa.hawaii.edu

Voslářová E, and Passantino A (2012). Stray dog and cat laws and enforcement in Czech Republic and in Italy. Annali Dell’Istituto Superiore Di Sanita, 48: 97-104. DOI: https://www.doi.org/10.4415/ann_12_01_16

World Organization for Animal Health (OIE). (2019). Stray dogs population control. Terrestrial Animal Health Code, pp. 1-12. Available at: http://www.oie.int/fileadmin/Home/eng/Health_standards/tahc/current/chapitre_aw_stray_dog.pdf 